\title{
Measurements of the linac coherent light source laser heater and its impact on the $x$-ray free-electron laser performance
}

\author{
Z. Huang, A. Brachmann, F.-J. Decker, Y. Ding, D. Dowell, P. Emma, J. Frisch, S. Gilevich, G. Hays, Ph. Hering, \\ R. Iverson, H. Loos, A. Miahnahri, H.-D. Nuhn, D. Ratner, G. Stupakov, J. Turner, J. Welch, W. White, J. Wu, and D. Xiang \\ SLAC National Accelerator Laboratory, Menlo Park, California 94025, USA
}

(Received 4 December 2009; published 17 February 2010)

\begin{abstract}
The very bright electron beam required for an x-ray free-electron laser (FEL), such as the linac coherent light source (LCLS), is susceptible to a microbunching instability in the magnetic bunch compressors, prior to the FEL undulator. The uncorrelated electron energy spread in the LCLS can be increased by an order of magnitude to provide strong Landau damping against the instability without degrading the FEL performance. To this end, a "laser-heater" system has been installed in the LCLS injector, which modulates the energy of a 135-MeV electron bunch with an IR-laser beam in a short undulator, enclosed within a four-dipole chicane. In this paper, we report detailed measurements of laser-heater-induced energy spread, including the unexpected self-heating phenomenon when the laser energy is very low. We discuss the suppression of the microbunching instability with the laser heater and its impact on the x-ray FEL performance. We also present the analysis of these experimental results and develop a threedimensional longitudinal space charge model to explain the self-heating effect.
\end{abstract}

\section{INTRODUCTION}

The linac coherent light source (LCLS) is an x-ray freeelectron laser (FEL) that has achieved its first lasing and saturation at $1.5 \AA$ radiation wavelength [1]. The very bright electron beam required for an $\mathrm{x}$-ray free-electron laser (FEL), such as the LCLS, is susceptible to a microbunching instability in the magnetic bunch compressors that may increase the slice energy spread beyond the FEL tolerance [2-7]. A laser heater [6,8] has been suggested to add a small level of energy spread to the electron beam in order to Landau damp the microbunching instability before it potentially breaks up the high-brightness electron beam. Such a system has been designed for the LCLS [7] and is now incorporated in almost all short-wavelength FEL projects.

The LCLS laser-heater system was fully installed and tested in the fall of 2008, and effects of heating on the electron beam and the $\mathrm{x}$-ray FEL were studied during the 2009 commissioning period. Preliminary results were reported in Ref. [9]. In this paper, we present detailed measurements of laser-heater-induced energy spread, including the unexpected self-heating phenomenon when the laser energy is very low. We discuss the suppression of the microbunching instability with the laser heater and its impact on the x-ray FEL performance. These measurements were performed with the nominal bunch charge of $250 \mathrm{pC}$ for the LCLS operation. We also discuss the analysis of these experimental results and develop a three-dimensional longitudinal space charge model to explain the self-heating effect.

\section{LASER-HEATER SETUP}

\section{A. Main parameters}

The LCLS laser heater (LH) is installed in the LCLS injector area at $135 \mathrm{MeV}$, right before the rf deflector that is used for the time-resolved electron diagnostics (see Fig. 1). The LH is composed of a 4-dipole chicane; a 10-period, planar, pure-permanent-magnet, adjustable-gap undulator located at the center of the chicane [10]; one optical transition radiation (OTR) screen on each side of the undulator for electron/laser spatial alignment; and an infrared (IR) laser (up to 20-MW power) which copropagates with

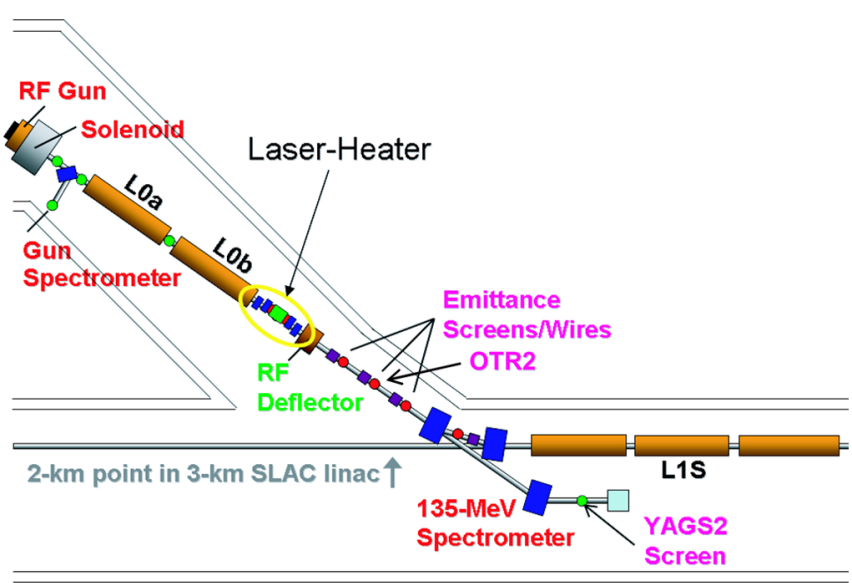

FIG. 1. (Color) The LCLS injector layout showing laser heater, transverse rf deflector, OTR/YAG screens, wire scanners, and spectrometers. 
TABLE I. Main parameters for the LCLS laser heater (LH) (at $135 \mathrm{MeV})$.

\begin{tabular}{lccc}
\hline \hline Parameter & Symbol & Value & Unit \\
\hline LH-undulator pole full gap & $g_{u}$ & 34.5 & $\mathrm{~mm}$ \\
LH-undulator parameter & $K$ & 1.38 & \\
LH-undulator period & $\lambda_{u}$ & 5.4 & $\mathrm{~cm}$ \\
Number of undulator periods & $N_{u}$ & 10 & \\
IR-laser wavelength & $\lambda_{L}$ & 758 & $\mathrm{~nm}$ \\
IR-laser energy (nominal 6 $\mu \mathrm{J})$ & $E_{L}$ & $<230$ & $\mu \mathrm{J}$ \\
IR-laser pulse duration (FWHM) & $T_{L}$ & $10-20$ & $\mathrm{ps}$ \\
Horizontal offset at chicane center & $\Delta x$ & 35 & $\mathrm{~mm}$ \\
Bend angle of each dipole & $|\theta|$ & 7.5 & $\mathrm{deg}$ \\
Chicane momentum compaction & $R_{56}^{T}$ & 7.8 & $\mathrm{~mm}$ \\
Electron rms transverse size & $\sigma_{x, y}$ & $\sim 150$ & $\mu \mathrm{m}$ \\
IR-laser rms spot size & $\sigma_{r}$ & $\sim 210$ & $\mu \mathrm{m}$ \\
Laser Rayleigh length & $Z_{R}$ & $\sim 70$ & $\mathrm{~cm}$ \\
\hline \hline
\end{tabular}

the electron beam inside the undulator generating a 758$\mathrm{nm}$ energy modulation along the bunch. The final two dipoles of the 4-dipole chicane time-smear this modulation leaving only a thermal-like intrinsic energy spread within the bunch. Table I lists the main parameters for this system.

\section{B. Optics and alignment}

The unconverted IR beam from the photocathode drive laser is guided by the IR beam transport optics from the laser room down to the accelerator tunnel (see Fig. 2). Laser diagnostics include two imaging cameras (VHC and $\mathrm{CH} 1$ ), power meter, photodiode for rough temporal overlapping, motorized mirrors (MH2 and $\mathrm{MH} 3$ ) for position feedback control, an adjustable delay line, a wave plate, an attenuator, and a shutter for energy control.

Two $1-\mu \mathrm{m}$ aluminum OTR screens are included with one on each side of the undulator in order to align the laser beam with respect to the electron beam transversely and to measure the beam sizes. Since these thin OTR screens have shown damage with $>2 \mu \mathrm{J}$ of IR energy at $10 \mathrm{~Hz}$ beam

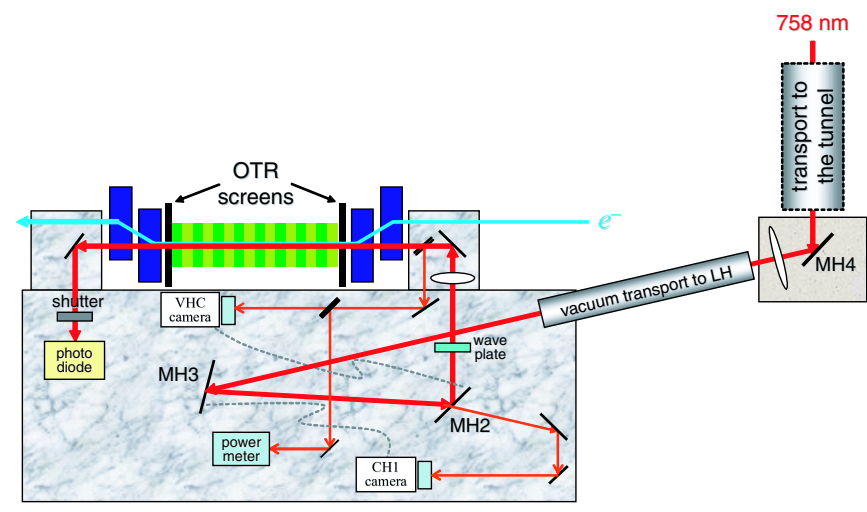

FIG. 2. (Color) The layout of the laser-heater system showing LH chicane, undulator, OTR screens, laser transport, motorized mirrors, cameras, wave plate, power meter, and photodiode. repetition rate, they are protected from IR-laser damage by logic which drops in a laser attenuator when either of the OTR screens are inserted. Figure 3 shows the individual images of the laser light and the optical radiation signal generated by the electron beam on the two OTR screens. The "electron" image on OTRH1 (the first OTR screen) shows both the OTR signal and synchrotron radiation from the second dipole [Fig. 3(c)], while the electron image on OTRH2 (the second OTR screen) shows the OTR signal together with undulator radiation pattern [Fig. 3(d)]. The four lobes are the vertical polarization component of the fundamental undulator radiation. The motorized mirrors $\mathrm{MH} 2$ and $\mathrm{MH} 3$ adjust the offset positions of the laser centroid relative to the electron centroid until within $\sim 100 \mu \mathrm{m}$ tolerance level. The transverse alignment process has been automated using a MATLAB-based program.

The IR-laser pulse length is stretched by a pair of gratings to a FWHM pulse duration from 10 to $20 \mathrm{ps,} \mathrm{while}$ the electron bunch length in the injector is typically 5 to $6 \mathrm{ps}$ (FWHM) for the nominal charge of $250 \mathrm{pC}$. A streak camera measurement of the laser temporal profile is shown in Fig. 4. A fast photodiode is used to sense the electron arrival time using the undulator radiation signal and to establish coincidence with the laser pulse within $10 \mathrm{ps}$. Finer timing adjustment can be made by using the laser delay line in the laser room with remote control.

\section{MEASUREMENT OF HEATING EFFECTS}

\section{A. Energy spread measurements}

When properly aligned in space and time, the laserelectron interaction will generate significant energy spread that is easily detected using the $135-\mathrm{MeV}$ spectrometer shown in Fig. 1. The spectrometer is designed to have a very large dispersion $\left(\left|\eta_{s}\right| \approx 0.9 \mathrm{~m}\right)$ and a small beta function ( $\beta \approx 1 \mathrm{~m}$ ) on a yttrium aluminum garnet (YAG) screen ("YAGS2" in Fig. 1) in order to have a few keV energy resolution. Figure 5 shows the measured beam profiles on YAGS2 for three different IR-laser pulse energy settings $(0,10 \mu \mathrm{J}$, and $220 \mu \mathrm{J})$. The transverse rf deflector (Fig. 1) is switched on here converting the vertical axis on YAGS2 to time (the bunch length coordinate), while the $135-\mathrm{MeV}$ spectrometer bend converts the horizontal YAGS2 axis to energy. Thus, the beam profiles on YAGS2 correspond to the longitudinal phase space of the electron bunch and clearly demonstrate that the time-sliced energy spread is increased with the IR laser. By zooming into a thin time slice of YAGS2 profile, the slice energy distribution and the rms energy spread can be obtained as shown in Fig. 6 (at the maximum LH energy). The laser rms transverse size in the middle of the undulator can be imaged by the VHC camera (Fig. 2) and is about $210 \mu \mathrm{m}$ as shown in Fig. 7, while the electron beam size is about $150 \mu \mathrm{m}$ based on the OTRH1 image. Thus, the laser spot size is somewhat larger than the electron spot size, resulting in the double-horn energy distribution [Fig. 6(a)]. The 


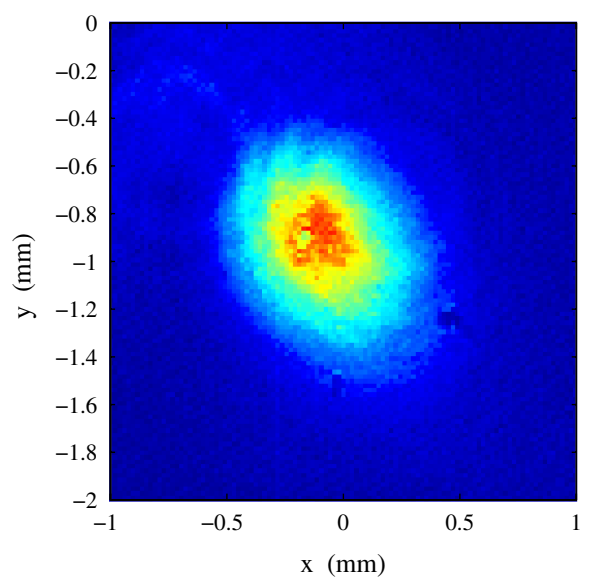

(a) OTRH1 laser image

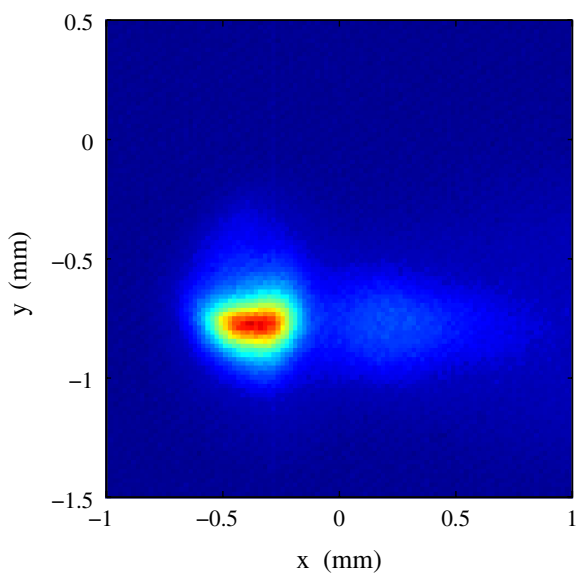

(c) OTRH1 "electron" image

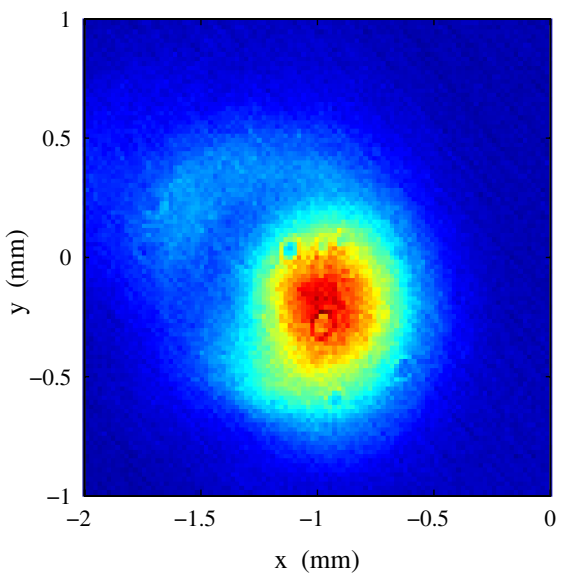

(b) OTRH2 laser image

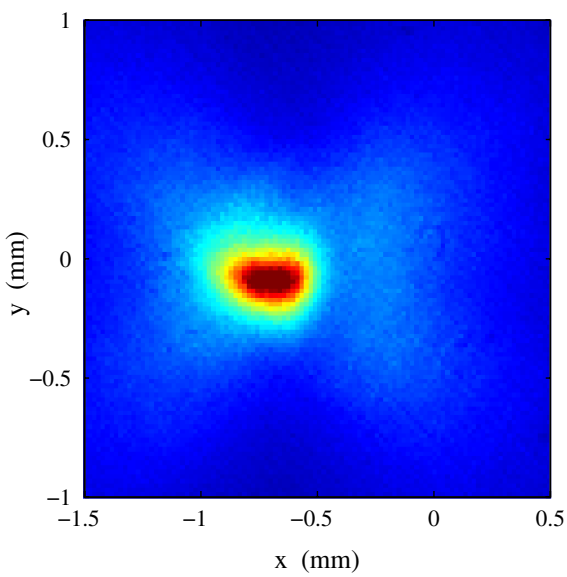

(d) OTRH2 "electron" image

FIG. 3. (Color) Beam images on LH OTR screens (OTRH1 and H2) before transverse alignment, see text for details.

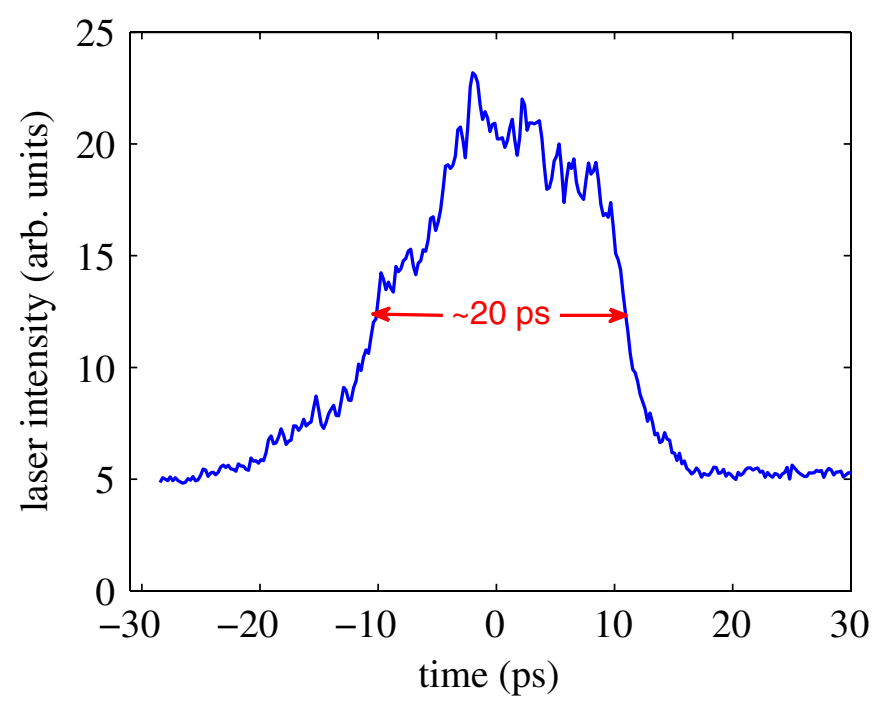

FIG. 4. (Color) An example of the heater laser temporal profile measured on a streak camera. The laser FWHM pulse duration is about $20 \mathrm{ps,}$ and the high-frequency temporal structures are due to the measurement noise. energy distribution is more Gaussian-like when the laser matches electron beam size in the undulator [Fig. 6(b)] and is determined to be more effective in suppressing microbunching instability [7]. However, in order to overcome position jitter that may misalign the electron beam relative to the laser spot, we normally choose to have a slightly larger laser spot size than that of the electrons.

Figure 8 shows the measured central slice rms energy spread as a function of the LH energy. In comparison, theoretical estimations based on the following argument are used. When the electron and the laser transverse sizes do not change much in the undulator, the maximum energy modulation amplitude induced by the laser-electron interaction is given by Eq. (8) of Ref. [7], and the rms energy spread is approximately

$$
\sigma_{\Delta E} \approx \sqrt{\frac{\sigma_{r}^{2}}{2\left(\sigma_{x}^{2}+\sigma_{r}^{2}\right)}} \sqrt{\frac{P_{L}}{P_{0}}} \frac{K[\mathrm{JJ}] N_{u} \lambda_{u} m c^{2}}{\gamma_{0} \sigma_{r}},
$$

where $P_{L}$ is the peak laser power, $P_{0}=8.7 \mathrm{GW},[\mathrm{JJ}]$ is the usual Bessel function factor associated with a planar undulator, $N_{u}$ is the number of full undulator periods (see Table I), $\gamma_{0} m c^{2}=135 \mathrm{MeV}$ is the injector energy, 


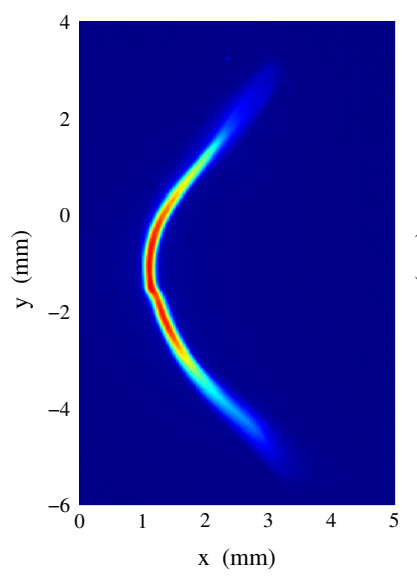

(a) no heating

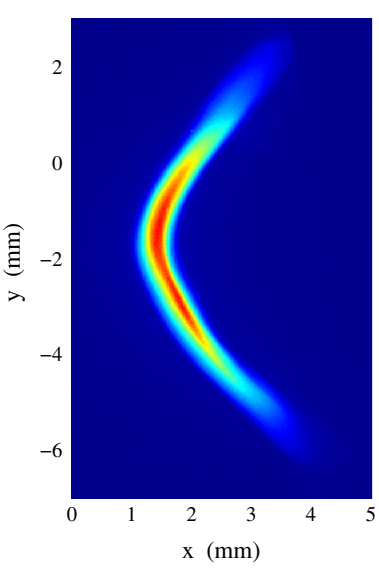

(b) nominal heating

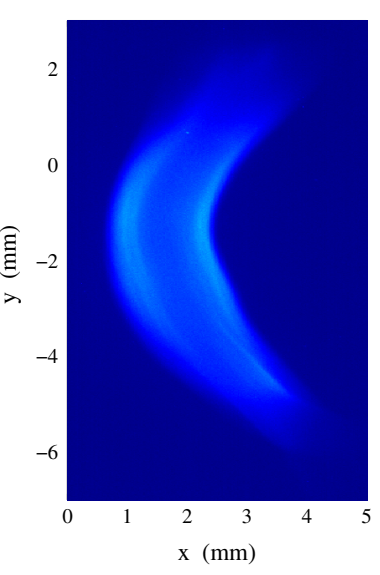

(c) maximum heating

FIG. 5. (Color) Measured longitudinal phase space on "YAGS2" screen at $135 \mathrm{MeV}$ with (a) laser heater off, (b) IR-laser energy at $10 \mu \mathrm{J}$, and (c) at $220 \mu \mathrm{J}$.

$\sigma_{x}=150 \mu \mathrm{m}$ is the rms electron transverse size, and $\sigma_{r}=210 \mu \mathrm{m}$ is the rms laser spot size in the undulator. The peak laser power is estimated based on the measured laser energy and the measured FWHM pulse length as shown in Fig. 4. We also perform ELEGANT [11] tracking using these parameters and present the simulation results in Fig. 8.

When the laser-heater shutter is closed, the measured slice energy spread is about $8-10 \mathrm{keV}$, limited by the horizontal beam size and YAG screen resolution. In addition, the rf deflecting cavity can generate a few $\mathrm{keV}$ slice energy spread due to the transverse dependence of its longitudinal field [12]. The intrinsic slice energy spread
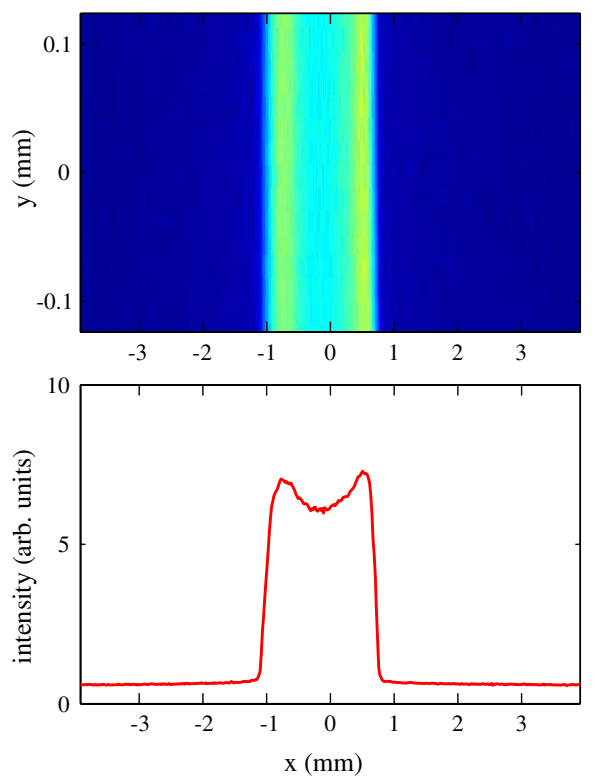

(a) Larger laser spot from injector simulations is around 2 to $3 \mathrm{keV}$. Nevertheless, for a very small amount of LH energy $(<5 \mu \mathrm{J})$, we observe a sudden increase of heating effect on YAGS2 (the bump region in Fig. 8 at low heater energy) that is not consistent with Eq. (1) and ELEGANT tracking. We will discuss this anomalous heating effect in Sec. III D.

\section{B. Undulator gap variation}

The LH-undulator gap can be controlled remotely to change the undulator magnetic field and hence the resonant condition. To clearly see the laser effect when the gap is out of resonance, we subtract in quadrature the minimum beam size on YAGS2 when the laser is off from the measured
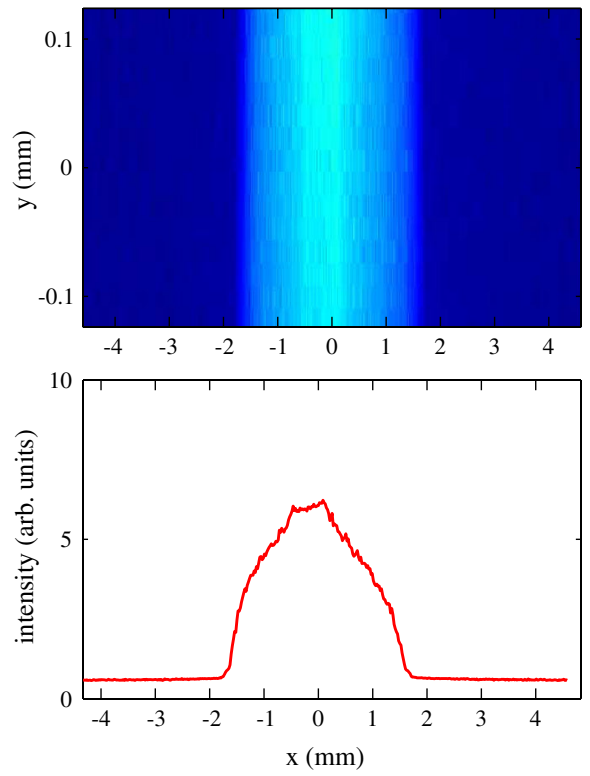

(b) Matched laser spot

FIG. 6. (Color) Central slice images (upper plots) and horizontal profiles (lower plots) showing both the double-horn (a) and more Gaussian-like (b) energy distributions. 

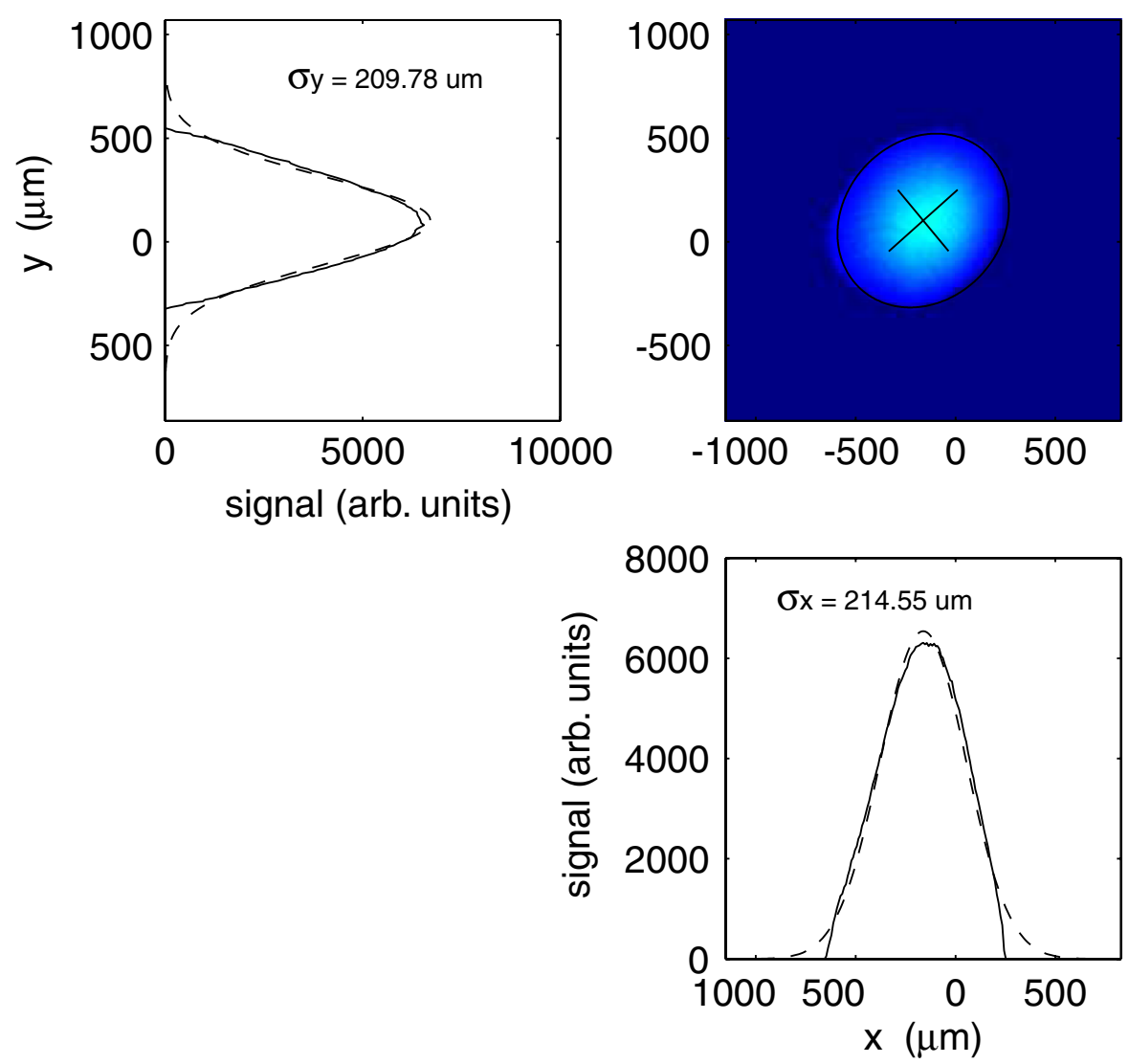

FIG. 7. (Color) The laser spot in the middle of the undulator as imaged by the VHC camera. The solid curves are horizontal ( $x$ ) and vertical $(y)$ projections of the image, and the dashed curves are the Gaussian fit for comparison. The base of the image is truncated by the software.

beam size when the laser energy is set at $200 \mu \mathrm{J}$ (Fig. 9). The resonant interaction of the laser and the electron beam is achieved at $g_{u} \approx 34 \mathrm{~mm}$, with the full resonance width of $\sim 2 \mathrm{~mm}$ in gap. In order to compare with the theory and simulations, we use a fitting formula between the undulator

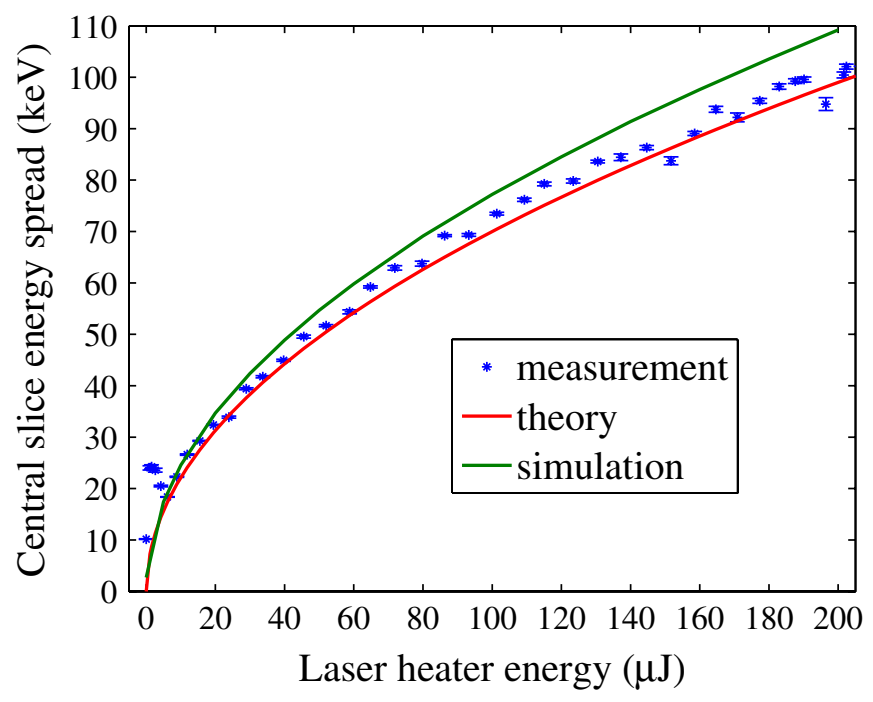

FIG. 8. (Color) Central slice rms energy spread vs LH energy. parameter $K$ and the gap $g_{u}$ (in $\mathrm{mm}$ ) as

$$
K\left(g_{u}\right)=9.527 \exp \left(-\frac{g_{u}}{17.66785}\right)
$$

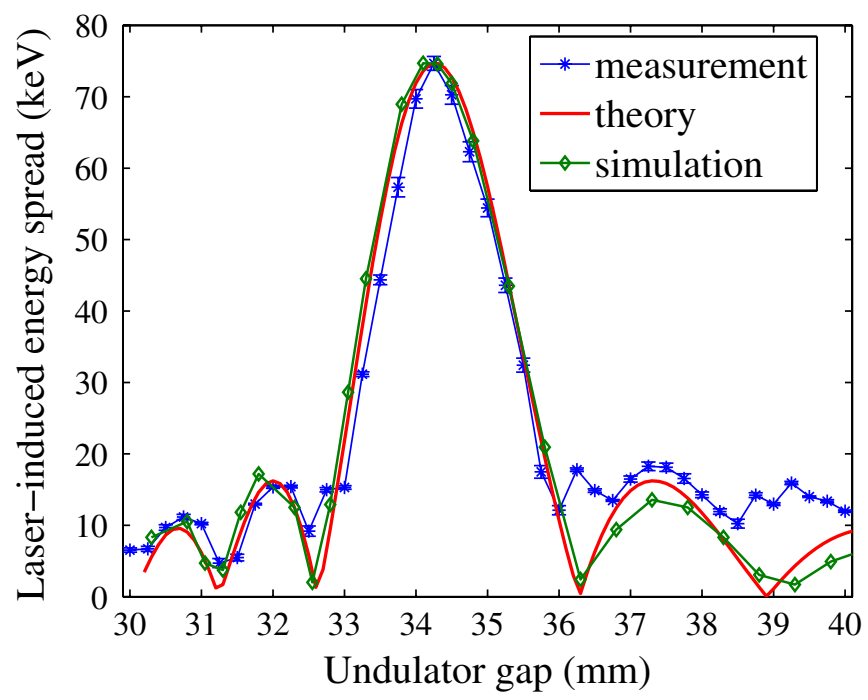

FIG. 9. (Color) Laser-induced rms slice energy spread vs LHundulator gap (LH energy is about $200 \mu \mathrm{J}$ ). 
This formula fits the measured undulator magnetic field data to a few percent within the gap variation range. The laser-beam interaction for a detuned undulator gap is given by

$$
\sigma_{\Delta E}\left(g_{u}\right)=\left(\sigma_{\Delta E}\right)_{\max }\left|\frac{\sin \left\{\pi N_{u}\left[\lambda\left(g_{u}\right)-\lambda_{L}\right] / \lambda_{L}\right\}}{\pi N_{u}\left[\lambda\left(g_{u}\right)-\lambda_{L}\right] / \lambda_{L}}\right|,
$$

where

$$
\lambda\left(g_{u}\right)=\frac{\lambda_{u}}{2 \gamma_{0}^{2}}\left[1+\frac{K\left(g_{u}\right)^{2}}{2}\right]
$$

In Fig. 9, the theoretical curve and ELEGANT simulation results are shifted by $+0.2 \mathrm{~mm}$ in terms of the undulator gap to fit the observed resonance. This shift may be caused by a slight miscalibration of the gap distance or a shift of the laser wavelength.

\section{Effects on transverse emittance}

The induced energy spread at the center of the chicane may cause some horizontal emittance growth. This can be estimated by comparing the heater-induced energy spread, $\sigma_{\delta}$, multiplied by the dispersion at the center of the chicane, $\eta_{c}$, to the nominal beam size, $\sigma_{x}$, or

$$
\frac{\Delta \epsilon_{x}}{\epsilon_{x}} \approx \frac{1}{2}\left(\frac{\sigma_{\delta} \eta_{c}}{\sigma_{x}}\right)^{2}
$$

At the full heater energy of $250 \mu \mathrm{J}, \sigma_{\delta}=\sigma_{\Delta E} / E=$ $0.12 / 135 \approx 9 \times 10^{-4}, \quad \eta_{c}=-35 \mathrm{~mm}, \quad$ and $\sigma_{x} \approx$ $150 \mu \mathrm{m}$, the relative emittance growth is extremely small (i.e., $\Delta \epsilon_{x} / \epsilon_{x}<2 \%$ ). Figure 10 shows the measured slice emittance on OTR2 for three different laser-heater settings. The measurements are carried out using the downstream rf deflector in combination with the quad scan method on

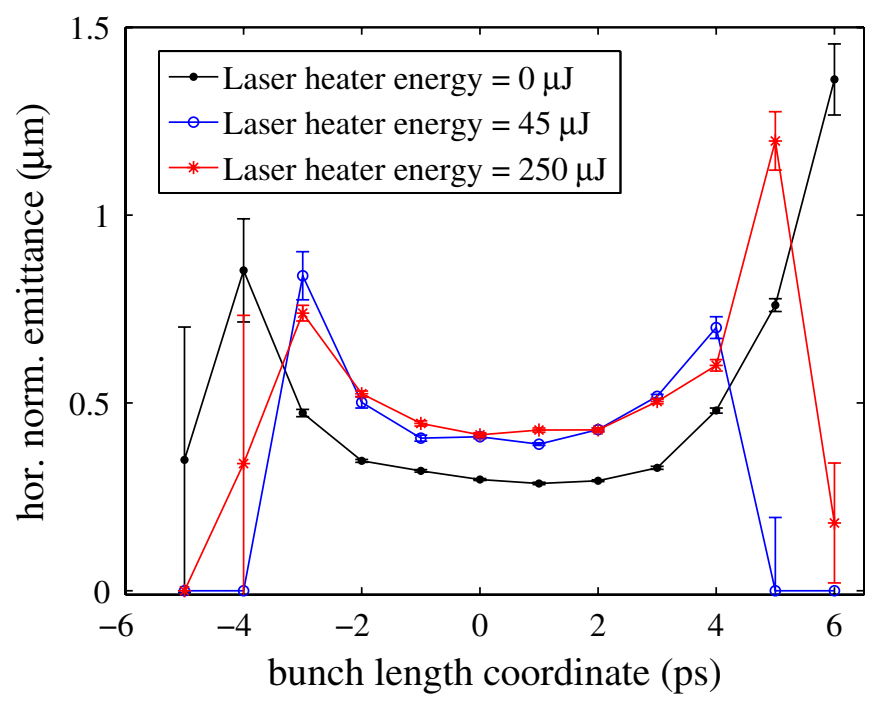

FIG. 10. (Color) Measured horizontal slice emittance at $250 \mathrm{pC}$ bunch charge on OTR2 vs bunch coordinate for different laserheater energies.
OTR2 (see Fig. 1). When the laser is turned off (i.e., laser energy at $0 \mu \mathrm{J}$ ), the LH chicane with $R_{56}^{T}=7.8 \mathrm{~mm}$ can convert any residual energy modulation in the injector to a small level of density modulation that causes the presence of coherent optical transition radiation (COTR) on the OTR2 screen (see Fig. 1). This effect makes the OTR2 emittance measurements inaccurate when the laser is off. Turning on the laser heater can easily suppress the small level of COTR on OTR2. As shown in Fig. 10, the slice emittance in the core part of the beam remains almost the same ( $\sim 0.4 \mu \mathrm{m}$ with $250 \mathrm{pC}$ of bunch charge) when the laser energy is increased from $45 \mu \mathrm{J}$ to $250 \mu \mathrm{J}$. This confirms that the modest level of laser heating does not increase the slice emittance of the electron beam.

\section{Trickle heating}

We now zoom into the bump region of Fig. 8 and perform a finer scan of the laser energy with the help of a preattenuator that limits the maximum laser energy to about $18 \mu \mathrm{J}$. Figure 11 shows the measured data (blue dots) and the result of Eq. (1) for a 20-ps FWHM laser pulse (black line). We now refer to the anomalous increase of slice energy spread at very low laser energies as "trickle heating." The peak of the trickle heating is around $1 \mu \mathrm{J}$ laser energy. From the black line in Fig. 11, the laserinduced rms energy spread is expected to be $7.5 \mathrm{keV}$, while the experimental result shows an rms energy spread of $28 \mathrm{keV}$. The laser-induced energy modulation can be converted to the longitudinal density modulation through $R_{56}=R_{56}^{T} / 2=3.9 \mathrm{~mm}$ of the half LH chicane, and an energy deviation of $7.5 \mathrm{keV}$ yields a longitudinal position shift of about a quarter laser wavelength. Thus, the longitudinal density modulation after the LH chicane is maximized around the laser energy corresponding to the trickle heating peak and can potentially drive collective effects to

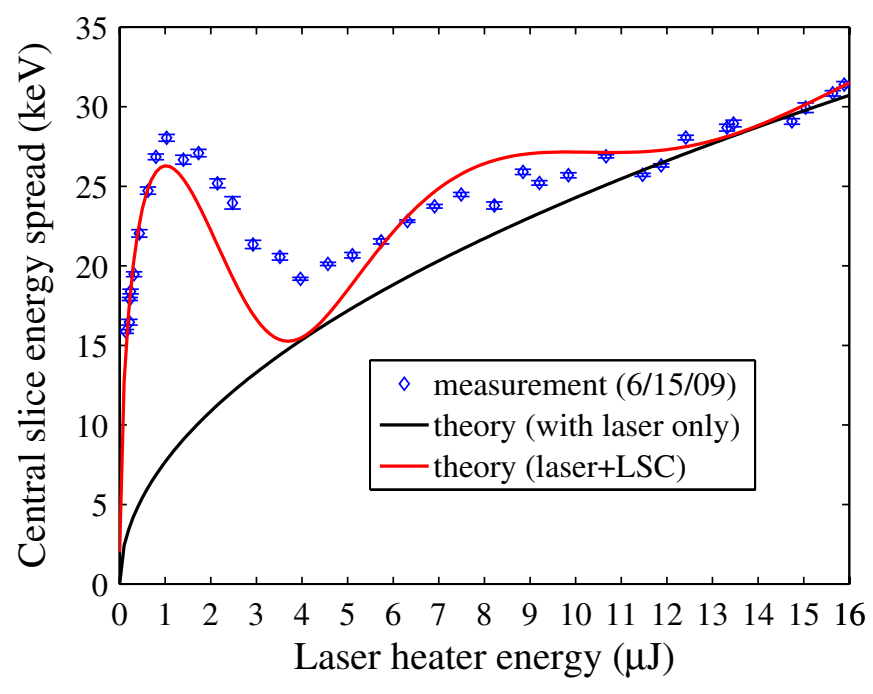

FIG. 11. (Color) Central slice rms energy spread vs LH energy in the trickle heating region. 


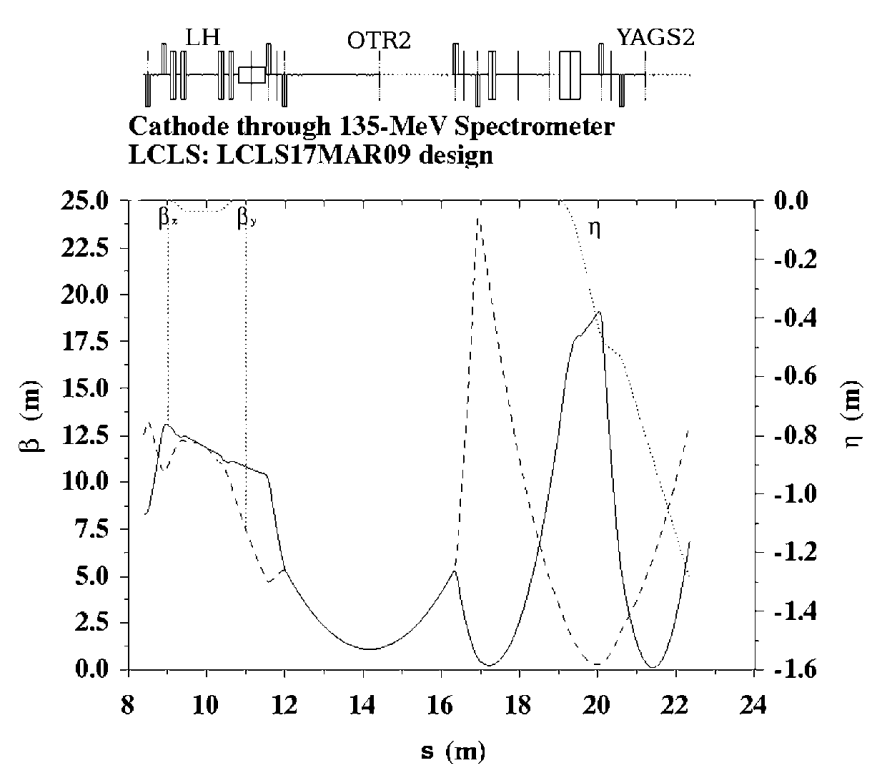

FIG. 12. Designed beta and dispersion functions from the laser heater to $135-\mathrm{MeV}$ spectrometer in the LCLS injector.

further increase the slice energy spread. Nevertheless, the observed large energy spread increase is still surprising because the longitudinal density modulation is mostly smeared out by the angular divergence of the electron beam and the nonzero $R_{52}=-\eta_{c}=35 \mathrm{~mm}$ from the chicane center (where the laser heater is located) to the chicane end. One-dimensional models of coherent synchrotron radiation in bends and longitudinal space charge (LSC) in straight sections downstream of the laser heater yield negligible energy spread increase and hence cannot explain the magnitude of the trickle heating. Further analysis illustrates that the beam density modulation is not truly smeared out by $R_{52}$ of the half chicane. Instead, the modulation is hidden in the $x^{\prime}-z$ plane, where $x^{\prime}$ is the electron horizontal angle, and $z$ is the longitudinal coordinate. After the beam propagates in the downstream straight section (see Fig. 12) for about $90^{\circ}$ betatron phase advance, this $x^{\prime}-z$ correlation develops into $x-z$ correlation as shown in Fig. 13. In the Appendix, a three-dimensional analysis demonstrates that a high-brightness LCLS beam with microbunching titled in the $x-z$ plane may still generate strong energy modulation due to the LSC interaction, especially when the electron transverse size is very small in the beam waist region in Fig. 12 (around $s=14 \mathrm{~m}$ ). In such a case, the total energy spread is approximately given by

$$
\sigma_{\delta_{f}}=\sqrt{\sigma_{\delta 0}^{2}+\left(\frac{\sigma_{\Delta E}}{\gamma_{0} m c^{2}}\right)^{2}+2\left|\delta_{\mathrm{LSC}}\right|^{2}}
$$

where $\sigma_{\delta 0}=2 / 135000=1.5 \times 10^{-5}$ is the initial slice energy spread, $\sigma_{\Delta E}$ is the laser-induced energy spread according to Eq. (1), and $2\left|\delta_{\mathrm{LSC}}\right|$ is the LSC-induced energy modulation amplitude according to Eq. (A28). In the theory (laser + LSC) curve shown in Fig. 11, we use

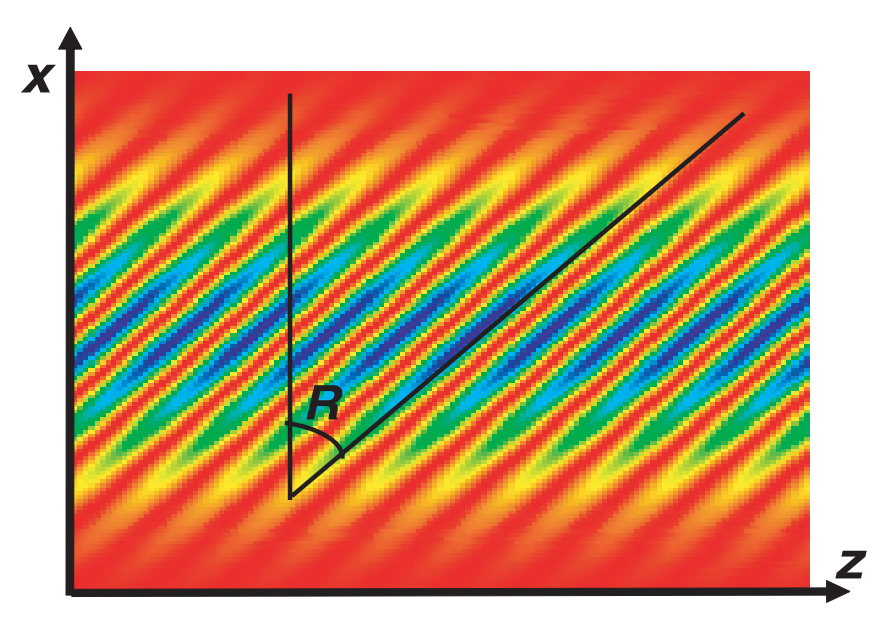

FIG. 13. (Color) A bunch with its density modulation tilted in the $x-z$ plane. The tilt angle is denoted as $R$, and the bunch head is to the right. Blue represents higher density region and red represents lower density region.

$\gamma \varepsilon=0.4 \mu \mathrm{m}, I_{0}=37 \mathrm{~A}$, and the optics functions from the beginning of the LH undulator to YAGS2 in order to integrate the accumulated LSC effect. The analytical result based on Eq. (6) agrees reasonably well with the measured data. In passing, we note that the effect of the trickle heating may be minimized by changing the optics downstream of the LH but has not been studied systematically yet.

\section{SUPPRESSION OF MICROBUNCHING INSTABILITY}

\section{A. Effects on COTR}

Coherent optical transition radiation (COTR) attributed to beam microbunching has been discovered during the LCLS commissioning $[13,14]$ and in other facilities as well $[15,16]$. This effect compromises the quantitative use of the many OTR screens downstream of the first bunch compressor. The laser heater suppresses these coherent signals by orders of magnitude in many cases but does not appear to completely remove a small level of COTR after compression. Figure 14 shows the intensity of OTR22 (an OTR screen right after the second bunch compressor) as a function of the laser-heater energy up to $\sim 250 \mu \mathrm{J}$. We see that the COTR intensity drops sharply from its maximum (when the laser-heater energy is close to $0 \mu \mathrm{J}$ ) and then starts to flatten out with the increasing LH energy. Further studies show that the COTR intensity on OTR22 at the full laser energy is still a factor of $\sim 5$ above the incoherent level and is hence too large to allow beam profile measurements. This nonexponential decay of the COTR intensity may be explained by the non-Gaussian energy profiles created by the laser heating (see Fig. 6), or it may be caused by a small part of the electron beam that escapes heating due to nonperfect temporal and spatial overlapping. As a result, alternative diagnostics such as 


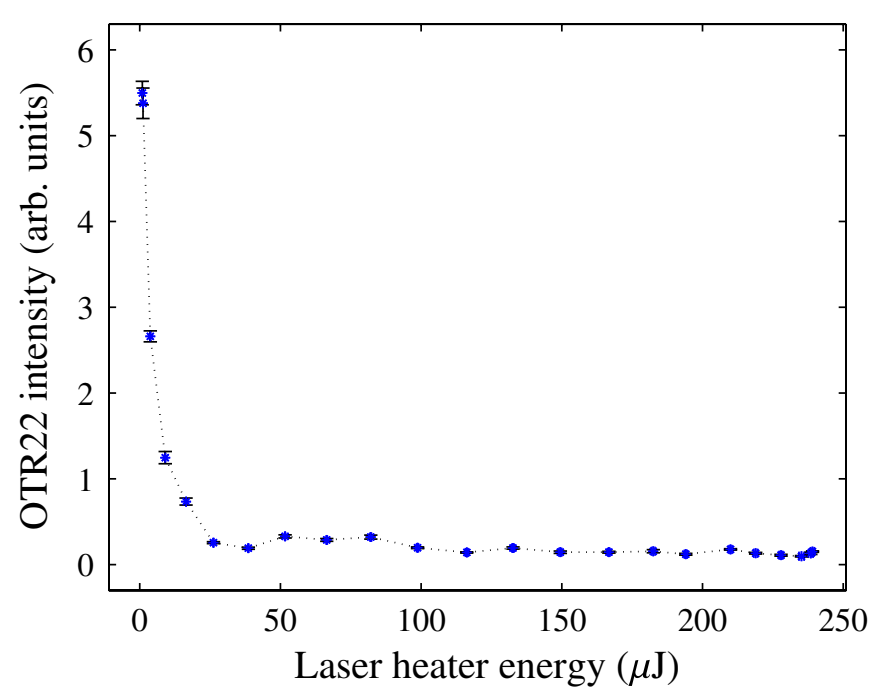

FIG. 14. (Color) COTR intensity on OTR22 (an OTR screen right after the second bunch compressor) vs LH energy.

wire scanners for electron beam size measurements are routinely used instead of any of the OTR screens that are located after the first bunch compressor in the LCLS.

\section{B. Effects on FEL}

LCLS first lasing and saturation at the FEL wavelength of $1.5 \AA$ have been reported in Ref. [1]. In this section, we illustrate the effects of the laser heater on the hard x-ray FEL performance. Figure 15 shows the effect of laser heater on the FEL intensity measured on a YAG screen downstream of the undulator beam line. To make a sensitive measurement with respect to electron energy spread, only 12 undulator sections (each $3.35 \mathrm{~m}$ in length) are inserted in the beam path to make sure the FEL is still in

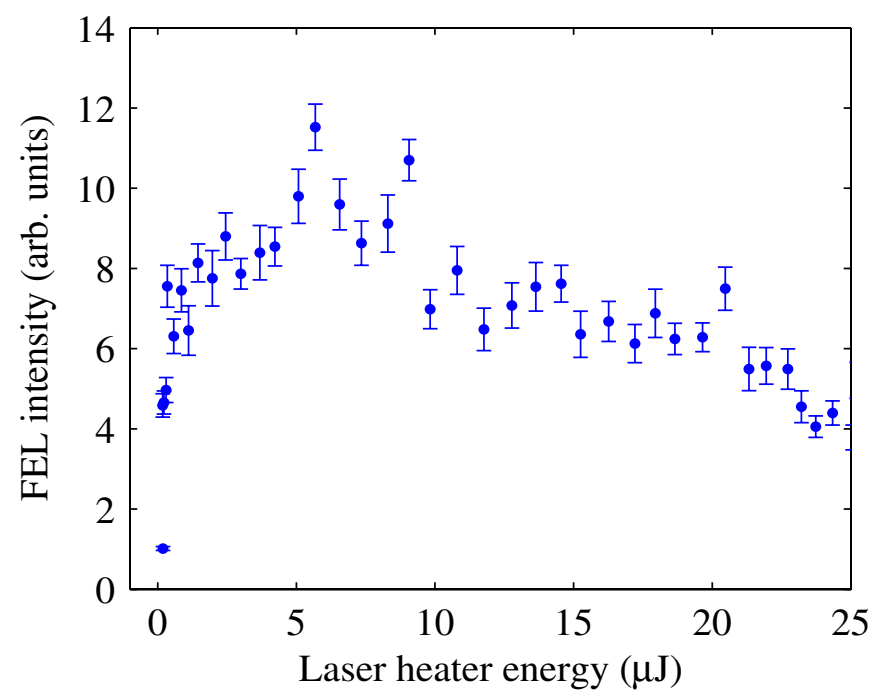

FIG. 15. (Color) FEL intensity at $1.5 \AA$ measured on a downstream YAG screen vs LH energy when 12 undulator sections are inserted. the exponential growth regime. The FEL power maximizes at the laser-heater setting of 6-7 $\mu \mathrm{J}$ and drops by an order of magnitude when the laser heater is turned off. For this reason, the laser-heater energy is normally set at $\sim 6 \mu \mathrm{J}$, corresponding to about $20 \mathrm{keV}$ rms energy spread measured on YAGS2 (slightly above the trickle heating region in Figs. 8 and 11). After going through two bunch compressors, the electron peak current is increased from $\sim 33 \mathrm{~A}$ to about $3 \mathrm{kA}$ with a total compression ratio of $3000 / 33 \approx 90$. Thus the slice energy spread becomes $20 \times 90=1.8 \mathrm{MeV}$, or a relative energy spread $1.3 \times$ $10^{-4}$ at $13.64 \mathrm{GeV}$.

FEL gain length can be measured by determining the FEL intensity as a function of the undulator length. The detailed gain length measurement methods are described in Ref. [17]. Figure 16 shows the measured FEL gain length at $1.5 \AA$ as a function of LH-induced rms energy spread. To compare with the theoretical expectations, we multiply this energy spread by the bunch compression factor $(\sim 90$ for $3 \mathrm{kA}$ final peak current) to estimate the final energy spread. We then apply Xie's gain length formula [18] for a normalized emittance of 0.4 and $0.5 \mu \mathrm{m}$, respectively. As shown in Fig. 16, the theoretical curves are consistent with the experimental data except when the heater is off. In fact, the measured gain length with the heater off $(0 \mathrm{keV})$ is higher than the nominal heater value $(20 \mathrm{keV})$ by about $1 \mathrm{~m}$, apparently due to the additional energy spread increase caused by the microbunching instability (not included in Xie's formula). The effective energy spread corresponding to this gain length is about $4.5 \mathrm{MeV}$ at the undulator entrance, about a factor of 2.5 higher than the estimated energy spread at the undulator entrance for the nominal laser-heater setting of $6 \mu \mathrm{J}$. This result suggests that the laser heater adequately controls the slice energy spread for the LCLS FEL.

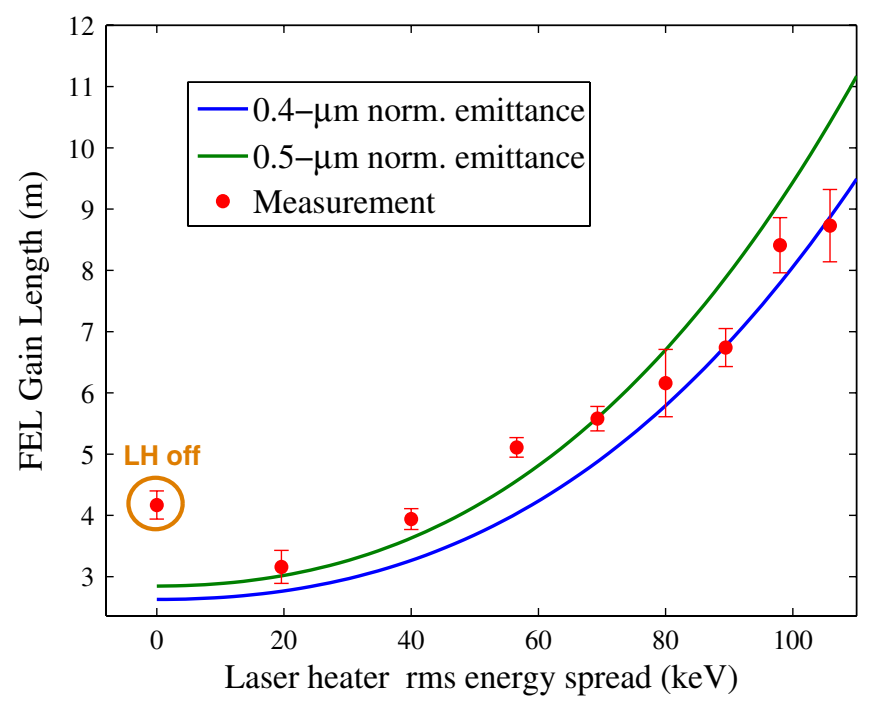

FIG. 16. (Color) FEL gain length at $1.5 \AA$ A vs LH-induced energy spread. 


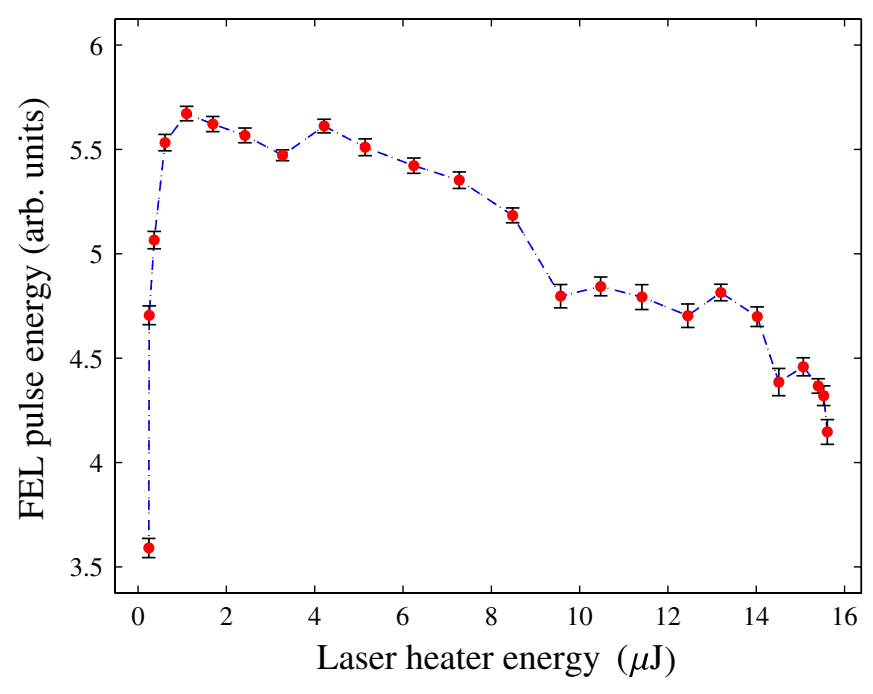

FIG. 17. (Color) FEL intensity at $1.5 \AA$ measured on a downstream YAG screen vs LH energy when all 33 undulator sections are inserted.

Finally, Fig. 17 shows the FEL intensity (measured on the same YAG screen as Fig. 15) as a function of laserheater energy when all 33 undulators are inserted in the beam path. The total undulator length is about $110 \mathrm{~m}$ while the FEL saturation length is about $60 \mathrm{~m}$ under the nominal LH setting [1]. Thus, the FEL is in the saturation regime and hence is not very sensitive to electron energy spread. In addition, the YAG screen signal may also reach saturation under such intense x-ray pulses. Nevertheless, the measured FEL intensity still drops by about a factor of 2 with LH off as compared to when the LH is set to the nominal value of $6 \mu \mathrm{J}$.

\section{Conclusions}

In summary, we show that the laser heater is successfully used in the LCLS to improve and optimize x-ray FEL performance at the nominal operating condition. Microbunching instability seems to be under adequate control for the FEL, but not all high-frequency structures on the electron beam are removed by the laser heater. As a result, there are still some levels of COTR after the first bunch compressor that contaminate OTR screens used for diagnostics. Alternative beam profile diagnostics such as wire scanners are used extensively in the LCLS and are essential for measuring such bright electron beams. In this paper, we also show how the unexpected trickle heating effect can be explained by a 3D LSC model. Although trickle heating does not affect the nominal LCLS operation, it may have implications to other laser-heater designs as well as laser manipulations of high-brightness beams.

\section{ACKNOWLEDGMENTS}

We thank the SLAC magnet measurement, engineering, controls, operations, and rf groups for their help and dedi- cated support. We also thank S. Spampinati for useful discussions and a careful reading of the manuscript. This work was supported by Department of Energy Contract No. DE-AC02-76SF00515.

\section{APPENDIX: LONGITUDINAL SPACE CHARGE FOR A BEAM WITH TILTED MICROBUNCHING}

\section{Three-dimensional analysis}

Consider a beam with the tilted microbunching as shown in Fig. 13. The beam can be described by a threedimensional (3D) density function as

$$
\begin{aligned}
\rho(x, y, z)= & \frac{N}{2 \pi \sigma_{x}^{2} L}\left\{1+2 a_{0} \cos \left[k_{0}(z-R x)\right]\right\} \\
& \times \exp \left(-\frac{x^{2}+y^{2}}{2 \sigma_{x}^{2}}\right),
\end{aligned}
$$

where $N$ is the total number of electrons, $\sigma_{x}$ is the transverse beam size, $L$ is the electron bunch length and is assumed to be long compared to the modulation wavelength, $2 a_{0}$ is the relative modulation amplitude at the modulation wave number $k_{0}$, and $R$ is the tilt angle which is related to the transfer matrix (see Fig. 13 and also the next section).

The longitudinal bunching factor at $k=k_{0}$ is given as

$$
\begin{aligned}
b\left(k_{0}\right) & =\frac{1}{N} \int_{-\infty}^{\infty} d x \int_{-\infty}^{\infty} d y \int_{-L / 2}^{L / 2} d z \rho(x, y, z) e^{-i k_{0} z} \\
& =a_{0} e^{-k_{0}^{2} \sigma_{x}^{2} R^{2} / 2} .
\end{aligned}
$$

When $\sigma_{x} R>1 / k_{0}$, the longitudinal density modulation is suppressed exponentially. In a one-dimensional (1D) impedance approach, the longitudinal space charge (LSC) field generated by this longitudinal modulation is

$$
E_{z}^{1 \mathrm{D}}\left(k_{0}\right)=-I_{0} b\left(k_{0}\right) Z_{1 \mathrm{D}}\left(k_{0}\right) \text {, }
$$

where $I_{0}=N e c / L$ is the beam current,

$$
\begin{aligned}
Z_{\mathrm{ID}}(k) & =\frac{i k Z_{0}}{2 \pi \gamma^{2}} \int \frac{r d r}{\sigma_{x}^{2}} \exp \left(-\frac{r^{2}}{2 \sigma_{x}^{2}}\right) K_{0}\left(\frac{k r}{\gamma}\right) \\
& =\frac{i k Z_{0}}{4 \pi \gamma^{2}} \exp \left(\frac{k^{2} \sigma^{2}}{2 \gamma^{2}}\right) \Gamma\left(0, \frac{k^{2} \sigma^{2}}{2 \gamma^{2}}\right)
\end{aligned}
$$

is the 1D LSC impedance per unit length for a transversely Gaussian beam, $Z_{0}$ is the vacuum impedance, $\gamma$ is the electron energy in units of rest mass energy $m c^{2}, K_{0}$ is the modified Bessel function, $r=\sqrt{x^{2}+y^{2}}$, and $\Gamma$ is the Euler gamma function. For $k \sigma_{x} / \gamma \gg 1$, we can take $\exp \left(-r^{2} / 2 \sigma_{x}^{2}\right)=1$ in Eq. (A4) to obtain

$$
Z_{1 \mathrm{D}}(k) \approx \frac{i Z_{0}}{2 \pi k \sigma_{x}^{2}} .
$$

Combining Eqs. (A2), (A3), and (A5), we obtain the 1D result 


$$
E_{z}^{1 \mathrm{D}}\left(k_{0}\right)=-i \frac{I_{0} Z_{0}}{2 \pi k_{0} \sigma_{x}^{2}} a_{0} \exp \left(-\frac{k_{0}^{2} R^{2} \sigma_{x}^{2}}{2}\right)
$$

It turns out this $1 \mathrm{D}$ result can underestimate the LSC effect by a large factor. For a proper 3D analysis, we first compute the LSC field with the Green function

$$
\begin{aligned}
E_{z}(x, y, z)= & \frac{e}{4 \pi \epsilon_{0}} \int d x_{1} d y_{1} d z_{1} \\
& \times \frac{\rho\left(x_{1}, y_{1}, z_{1}\right) \gamma\left(z-z_{1}\right)}{\left[\left(x-x_{1}\right)^{2}+\left(y-y_{1}\right)^{2}+\gamma^{2}\left(z-z_{1}\right)^{2}\right]^{3 / 2}} .
\end{aligned}
$$

The longitudinal Fourier transform of the on-axis $E_{z}(x=$ $0, y=0)$ is

$$
\begin{aligned}
E_{z}(k) & =\int_{-L / 2}^{L / 2} \frac{d z}{L} E_{z}(x=0, y=0, z) e^{-i k z} \\
& =\frac{-e i k}{2 \pi \epsilon_{0} \gamma^{2} L} \int d x_{1} d y_{1} d z_{1} \rho\left(x_{1}, y_{1}, z_{1}\right) e^{-i k z_{1}} K_{0}\left(\frac{k r}{\gamma}\right) .
\end{aligned}
$$

Putting Eq. (A1) into Eq. (A8), performing straightforward $z_{1}$ integration (which leads to $k=k_{0}$ ), and using $(r, \phi)$ instead of $\left(x_{1}, y_{1}\right)$ coordinates, we have

$$
\begin{aligned}
E_{z}\left(k_{0}\right)= & a_{0} \frac{-e i k_{0} N}{2 \pi \epsilon_{0} \gamma^{2} L} \int \frac{r d r}{2 \pi \sigma_{x}^{2}} \\
& \times \int_{0}^{2 \pi} d \phi \exp \left(-\frac{r^{2}}{2 \sigma_{x}^{2}}\right) e^{-i k_{0} R r \sin \phi} K_{0}\left(\frac{k r}{\gamma}\right) .
\end{aligned}
$$

We can expand

$$
e^{-i k_{0} R r \sin \phi}=\sum_{n=-\infty}^{\infty} J_{n}\left(-k_{0} R r\right) e^{i n \phi}
$$

using Bessel functions $J_{n}$. With scaled variables $\xi=$ $k_{0} r / \gamma$ and $\xi_{\sigma}=k_{0} \sigma_{x} / \gamma$, Eq. (A9) can be written as

$$
E_{z}\left(k_{0}\right)=a_{0} \frac{-e i k_{0} N}{2 \pi \epsilon_{0} \gamma^{2} L} \int \frac{\xi d \xi}{\xi_{\sigma}^{2}} \exp \left(-\frac{\xi^{2}}{2 \xi_{\sigma}^{2}}\right) J_{0}(\gamma R \xi) K_{0}(\xi) .
$$

In the limit when $\xi_{\sigma} \gg 1$, we take $\exp \left(-\xi^{2} / 2 \xi_{\sigma}^{2}\right)=1$ in Eq. (A11) to obtain

$$
E_{z}\left(k_{0}\right) \approx \frac{-i I_{0} Z_{0}}{2 \pi k_{0} \sigma_{x}^{2}} \frac{a_{0}}{1+\gamma^{2} R^{2}} .
$$

Thus, when $\sigma_{x} \gg \gamma / k_{0}$, the tilted microbunching does not suppress LSC exponentially, instead the suppression factor depends weakly on $\gamma R$. This weak dependence is similar to what was found in Ref. [19] when 3D aspects of LSC microbunching were included.
The ratio of Eq. (A9) and Eq. (A3) is

$$
\frac{E_{z}}{E_{z}^{1 \mathrm{D}}} \equiv F\left(\xi_{\sigma}, \gamma R\right)
$$

$F$ can be a very large number when $\xi_{\sigma}=k_{0} \sigma_{x} / \gamma>1$. In this regime, the 1D approach underestimates the LSC effect by a large factor. The $3 \mathrm{D}$ vs $1 \mathrm{D}$ ratio in this limit is

$$
F\left(\xi_{\sigma}, \gamma R\right)=\frac{e^{\gamma^{2} R^{2} \xi_{\sigma}^{2} / 2}}{1+\gamma^{2} R^{2}}=\frac{e^{k_{0}^{2} R^{2} \sigma_{x}^{2} / 2}}{1+\gamma^{2} R^{2}} .
$$

Let us take a numerical example close to the LCLS laserheater setup: $\lambda_{0}=2 \pi / k_{0}=758 \mathrm{~nm}, \quad \gamma=264, \quad \sigma_{x}=$ $60 \mu \mathrm{m}, \gamma R \sim 2$. Equation (A14) suggests that the $1 \mathrm{D}$ approach underestimates LSC by a factor of 250 .

\section{Applying to the LCLS trickle heating case}

When an electron passes through the LCLS laser heater, the longitudinal coordinate becomes

$$
z=z_{0}+R_{56}\left[\delta_{0}+\delta_{L} \sin \left(k_{0} z_{0}\right)\right]+R_{52} x_{0}^{\prime},
$$

where the subscript 0 refers to the location at the beginning of the laser-heater undulator, $R_{56}=3.9 \mathrm{~mm}, R_{52}=$ $-\eta_{c}=35 \mathrm{~mm}, R_{51}=0$ across the last half chicane. $\delta_{L}$ is the laser-induced relative energy modulation amplitude. For simplicity, we first assume that $\delta_{L}$ is independent of the transverse position of the electron beam $\left(x_{0}, y_{0}\right)$. This approximation is valid when the laser spot size is much larger than the electron spot size in the undulator (LCLS laser-heater laser spot size is about $40 \%$ larger than the electron spot size).

We wish to write Eq. (A15) in terms of the transverse coordinate $x$ and $x^{\prime}$ at an arbitrary location $s$ after the LH chicane. Since

$$
x=R_{11} x_{0}+R_{12} x_{0}^{\prime}, \quad x^{\prime}=R_{21} x_{0}+R_{22} x_{0}^{\prime} .
$$

Thus, we have

$$
x_{0}=R_{22} x-R_{12} x^{\prime}, \quad x_{0}^{\prime}=-R_{21} x+R_{11} x^{\prime} .
$$

Equation (A15) becomes

$$
z=z_{0}+R_{56}\left[\delta_{0}+\delta_{L} \sin \left(k_{0} z_{0}\right)\right]+\eta_{c}\left(R_{21} x-R_{11} x^{\prime}\right) .
$$

Note that $R_{21}$ and $R_{11}$ are functions of the beam line distance $s$, and $s=0$ refers to the beginning of the laserheater undulator. In terms of the Twiss parameters $\alpha(s)$ and $\beta(s)$, we have

$$
\begin{aligned}
& R_{11}(s)=\sqrt{\frac{\beta}{\beta_{0}}}\left(\cos \psi+\alpha_{0} \sin \psi\right), \\
& R_{21}(s)=\frac{\alpha_{0}-\alpha}{\sqrt{\beta \beta_{0}}} \cos \psi-\frac{1+\alpha \alpha_{0}}{\sqrt{\beta \beta_{0}}} \sin \psi,
\end{aligned}
$$

and the phase advance is 


$$
\psi(s)=\int_{0}^{s} \frac{d s^{\prime}}{\beta\left(s^{\prime}\right)} .
$$

We can now generalize Eq. (A8) to include integration over the $6 \mathrm{D}$ phase space distribution function $f$ as

$$
\begin{aligned}
E_{z}(k)= & \frac{-e i k}{2 \pi \epsilon_{0} \gamma^{2} L} \int d x d x^{\prime} d y d y^{\prime} d z d \delta f\left(x, x^{\prime}, y, y^{\prime}, z, \delta\right) \\
& \times e^{-i k z} K_{0}\left(\frac{k r}{\gamma}\right),
\end{aligned}
$$

where $\delta=\delta_{0}+\delta_{L} \sin \left(k_{0} z_{0}\right)$. Changing variables from $(z, \delta)$ to $\left(z_{0}, \delta_{0}\right)$, we can integrate over the longitudinal variables to obtain

$$
\begin{aligned}
E_{z}\left(k_{0}\right)= & \frac{i k_{0} I_{0} Z_{0}}{2 \pi \gamma^{2}} J_{1}\left(k_{0} R_{56} \delta_{L}\right) e^{-k_{0}^{2} R_{56}^{2} \sigma_{\delta 0}^{2} / 2} \\
& \times \int d x d x^{\prime} d y d y^{\prime} e^{-i k_{0} \eta_{c}\left(R_{21} x-R_{11} x^{\prime}\right)} f_{\perp}\left(x, x^{\prime}, y, y^{\prime}\right) \\
& \times K_{0}\left(\frac{k_{0} r}{\gamma}\right),
\end{aligned}
$$

where $J_{1}$ is the first-order Bessel function, $\sigma_{\delta 0}$ is the initial rms energy spread before the laser heater, and $f_{\perp}$ is the 4D transverse distribution function:

$$
\begin{aligned}
f_{\perp}\left(x, x^{\prime}, y, y^{\prime}\right)= & \frac{1}{(2 \pi \varepsilon)^{2}} \exp \left[-\frac{x^{2}+\left(\beta x^{\prime}+\alpha x\right)^{2}}{2 \varepsilon \beta}\right] \\
& \times \exp \left[-\frac{y^{2}+\left(\beta y^{\prime}+\alpha y\right)^{2}}{2 \varepsilon \beta}\right] .
\end{aligned}
$$

For simplicity, we have assumed that the Twiss parameters are the same in the vertical plane as in the horizontal plane, and $\varepsilon_{y}=\varepsilon_{x}=\varepsilon$ is the geometric emittance of the beam. In general, higher harmonic bunching of the laser frequency also exists just like in a high-gain harmonic generation FEL [20], but their effects are damped much stronger than the fundamental by beam energy spread and angular spread, and hence are neglected here.

Let us introduce new variables

$$
x_{1}^{\prime}=x^{\prime}+\frac{\alpha}{\beta} x, \quad y_{1}^{\prime}=y^{\prime}+\frac{\alpha}{\beta} y
$$

to replace $\left(x^{\prime}, y^{\prime}\right)$ in Eq. (A22). We can now integrate them out in Eq. (A22) to get

$$
\begin{aligned}
E_{z}\left(k_{0}\right) & =\frac{i k_{0} I_{0} Z_{0}}{2 \pi \gamma^{2}} J_{1}\left(k_{0} R_{56} \delta_{L}\right) \exp \left(-\frac{k_{0}^{2} R_{56}^{2} \sigma_{\delta 0}^{2}}{2}\right) \int d x d x_{1}^{\prime} d y d y_{1}^{\prime} e^{-i k_{0} \eta_{c}\left[\left(R_{21}+\alpha R_{11} / \beta\right) x-R_{11} x_{1}^{\prime}\right]} f_{\perp}\left(x, x_{1}^{\prime}, y, y_{1}^{\prime}\right) K_{0}\left(\frac{k r}{\gamma}\right) \\
& =\frac{i k_{0} I_{0} Z_{0}}{2 \pi \gamma^{2}} J_{1}\left(k_{0} R_{56} \delta_{L}\right) \exp \left(-\frac{k_{0}^{2} R_{56}^{2} \sigma_{\delta 0}^{2}}{2}-\frac{k_{0}^{2} \eta_{c}^{2} R_{11}^{2} \varepsilon}{2 \beta}\right) \int d x d y e^{-i k_{0} R x} \frac{1}{2 \pi \sigma_{x}^{2}} \exp \left(-\frac{x^{2}+y^{2}}{2 \sigma_{x}^{2}}\right) K_{0}\left(\frac{k r}{\gamma}\right),
\end{aligned}
$$

where $\sigma_{x}=\sqrt{\varepsilon \beta}$ is the rms transverse beam size at beam line position $s$, and

$$
R=\eta_{c}\left(R_{21}+\frac{\alpha}{\beta} R_{11}\right)
$$

We now recognize Eq. (A25) is similar to Eq. (A9) and can be readily integrated over $x$ and $y$. In the limit that $k \sigma_{x} / \gamma \gg 1$, the integration yields

$$
\begin{aligned}
E_{z}\left(k_{0}\right)= & \frac{i I_{0} Z_{0}}{2 \pi k_{0} \sigma_{x}^{2}} J_{1}\left(k_{0} R_{56} \delta_{L}\right) \\
& \times \exp \left(-\frac{k_{0}^{2} R_{56}^{2} \sigma_{\delta 0}^{2}}{2}-\frac{k_{0}^{2} \eta_{c}^{2} R_{11}^{2} \varepsilon}{2 \beta}\right) \frac{1}{1+\gamma^{2} R^{2}},
\end{aligned}
$$

Finally, we can integrate over the beam line distance $s$ from the chicane end to the observation point in order to obtain the LSC-induced energy modulation as

$$
\begin{aligned}
\delta_{\mathrm{LSC}}= & \int d s \frac{e E_{z}\left(k_{0}\right)}{\gamma m c^{2}} \\
= & \frac{2 i}{k_{0} \gamma} \frac{I_{0}}{I_{A}} J_{1}\left(k_{0} R_{56} \delta_{L}\right) \exp \left(-\frac{k_{0}^{2} R_{56}^{2} \sigma_{\delta 0}^{2}}{2}\right) \\
& \times \int d s \exp \left(-\frac{k_{0}^{2} \eta_{c}^{2} R_{11}^{2} \varepsilon}{2 \beta}\right) \frac{1}{\sigma_{x}^{2}\left(1+\gamma^{2} R^{2}\right)},
\end{aligned}
$$

where $I_{A}=e Z_{0} /\left(4 \pi m c^{2}\right)$ is the Alfvén current. Note that the real energy modulation amplitude is $2\left|\delta_{\mathrm{LSC}}\right|$.

In general, the laser-induced energy modulation depends on the initial transverse positions of the electron. For a Gaussian laser with the rms spot size $\sigma_{r}$, we have

$$
\delta_{L}\left(x_{0}, y_{0}\right)=\delta_{0} \exp \left(-\frac{x_{0}^{2}+y_{0}^{2}}{4 \sigma_{r}^{2}}\right) .
$$

Including this in Eq. (A15) makes the derivation much more complicated. To take into account this effect, we simply average $\delta_{L}$ over the transverse distribution at the laser heater as 


$$
\begin{aligned}
\bar{\delta}_{L} & =\left[\iint \frac{d x_{0} d y_{0}}{2 \pi \sigma_{x 0}^{2}} \delta_{L}^{2}\left(x_{0}, y_{0}\right) \exp \left(-\frac{x_{0}^{2}+y_{0}^{2}}{2 \sigma_{x 0}^{2}}\right)\right]^{1 / 2} \\
& =\delta_{0} \sqrt{\frac{\sigma_{r}^{2}}{\sigma_{x 0}^{2}+\sigma_{r}^{2}}}
\end{aligned}
$$

where $\sigma_{x 0}$ is the rms electron beam size at the heater center. $\bar{\delta}_{L}$ is the average energy modulation amplitude that yields the same laser-induced energy spread. It will be used in Eq. (A28) instead of $\delta_{L}$. Because of this averaging, we also assume that the LSC-induced energy modulation does not maintain any phase relationship with $\delta_{L}$, and the total energy spread is approximately given by

$$
\sigma_{\delta_{f}}=\sqrt{\sigma_{\delta 0}^{2}+\frac{\bar{\delta}_{L}^{2}}{2}+2\left|\delta_{\mathrm{LSC}}\right|^{2}}
$$

[1] P. Emma et al., in Proceedings of the 2009 Particle Accelerator Conference, Vancouver, BC, Canada.

[2] M. Borland et al., in Proceedings of the 2001 Particle Accelerator Conference, Chicago, Illinois, 2001 (IEEE, Piscataway, NJ, 2001), p. 2707; Nucl. Instrum. Methods Phys. Res., Sect. A 483, 268 (2002).

[3] E. Saldin, E. Schneidmiller, and M. Yurkov, Nucl. Instrum. Methods Phys. Res., Sect. A 490, 1 (2002).
[4] S. Heifets, G. Stupakov, and S. Krinsky, Phys. Rev. ST Accel. Beams 5, 064401 (2002).

[5] Z. Huang and K.-J. Kim, Phys. Rev. ST Accel. Beams 5, 074401 (2002).

[6] E. Saldin, E. Schneidmiller, and M. Yurkov, Nucl. Instrum. Methods Phys. Res., Sect. A 528, 355 (2004).

[7] Z. Huang et al., Phys. Rev. ST Accel. Beams 7, 074401 (2004).

[8] J. Galayda (private communication).

[9] P. Emma et al., Proceedings of the 2009 Particle Accelerator Conference (Ref. [1]).

[10] Z. Wolf, Report No. LCLS-TN-08-6, 2008.

[11] M. Borland, Report No. ANL/APS LS-287, 2000.

[12] D. Filippetto (private communication).

[13] R. Akre et al., Phys. Rev. ST Accel. Beams 11, 030703 (2008).

[14] H. Loos et al., Proceedings of the 2008 Free-Electron Laser Conference, Gyeongju, Korea, p. 485.

[15] A. Lumpkin et al., Phys. Rev. ST Accel. Beams 12, 080702 (2009).

[16] S. Wesch et al., in Proceedings of the 2009 Free-Electron Laser Conference, Liverpool, UK, p. 619.

[17] D. Ratner et al., Proceedings of the 2009 Free-Electron Laser Conference, Liverpool, UK, p. 221.

[18] M. Xie, Proceedings of the 1995 Particle Accelerator Conference (IEEE, Piscataway, NJ, 1995), p. 183.

[19] D. Ratner, A. Chao, and Z. Huang, Proceedings of the 2008 Free-Electron Laser Conference (Ref. [14]), p. 338.

[20] L. H. Yu, Phys. Rev. A 44, 5178 (1991). 